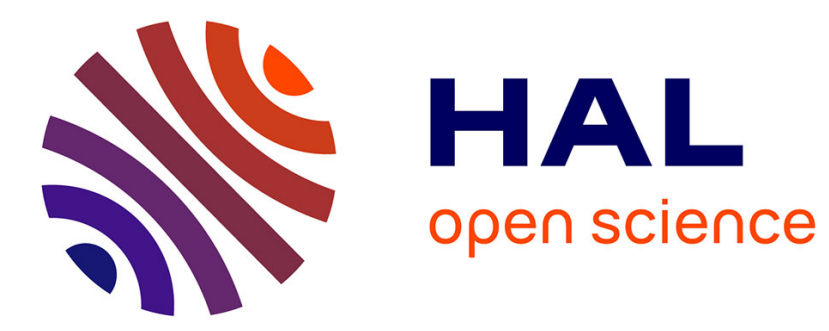

\title{
Anti-optimisation applied to the analysis of rotor/stator interaction
}

\author{
Tore Butlin, Alain Batailly
}

\section{To cite this version:}

Tore Butlin, Alain Batailly. Anti-optimisation applied to the analysis of rotor/stator interaction. Turbo Expo 2013, Jun 2013, San Antonio, United States. pp.GT2013-94633. hal-00833414

\section{HAL Id: hal-00833414 https://hal.science/hal-00833414}

Submitted on 12 Jun 2013

HAL is a multi-disciplinary open access archive for the deposit and dissemination of scientific research documents, whether they are published or not. The documents may come from teaching and research institutions in France or abroad, or from public or private research centers.
L'archive ouverte pluridisciplinaire HAL, est destinée au dépôt et à la diffusion de documents scientifiques de niveau recherche, publiés ou non, émanant des établissements d'enseignement et de recherche français ou étrangers, des laboratoires publics ou privés. 


\title{
Anti-optimisation applied to the analysis of rotor/stator interaction
}

Note: this article is part of the proceedings of the Turbo Expo 2013 conference (paper GT201394633).

\section{Tore Butlin}

RAEng / EPSRC Research Fellow

Cambridge University Engineering Department, Trumpington Street, Cambridge, CB2 1PZ, UK

\author{
Alain Batailly \\ Post-doctoral fellow \\ Structural Dynamics and Vibration Laboratory, Department of mechanical engineering, \\ McGill University, 817 Sherbrooke Ouest, Montréal, Québec H3A 0C3
}

\begin{abstract}
There is a drive towards minimising operating clearances within turbomachines in order to limit reverse leakage flows and hence improve their efficiency. This increases the likelihood of contact occurring between the blade and the casing, which can give rise to high amplitude vibration. Modelling this interaction represents a significantcomputational challenge. The non-linear contact precludes the use of well-established linear methods, and is also subject to uncertainties: the contact law is imprecisely known and the exact geometry of imperfections that trigger contact may be unknown.

In this paper a novel approach is presented that aims to account for the uncertainties associated with the non-linearity in a non-probabilistic way. The worst case is sought, by framing the system as a constrained anti-optimisation problem. The target to be maximised represents a metric of the output of interest. The degrees of freedom of the anti-optimisation are the non-linear input forces (considered as external loads), and the constraints are designed to capture what is thought to be known about the non-linear contact law and geometry.

A realistic three-dimensional model of a turbine blade is used to explore the approach, with contact considered at the leading and trailing edge. The blade dynamics are described in terms of a linear transfer function matrix and the target metric of interest is chosen to be the peak displacement of the contact points. The non-linearity is taken to result from an offset shaft, giving a sinusoidal clearance variation. The blade is driven at constant frequency and the scope of the study is limited to finding bounds on periodic solutions. A variety of constraint conditions are explored that describe aspects of the non-linearity. For example, only compressive forces are permitted (no tension from the contact), and the displacement must not exceed the clearance.

The method yields encouraging initial results: constraints can be identified that give efficient estimates of the upper bound response of the system as a function of drive frequency. The results are compared with a benchmark time-domain simulation and are found to correctly over-predict the response without being overly conservative. Broad trends are also in agreement with the benchmark solution. The proposed method appears to be a promising approach for efficiently accounting for uncertainties associated with the non-linearity and thus improving blade design.
\end{abstract}




\section{INTRODUCTION}

The demand for improved efficiency of aircraft engines places increasingly stringent requirements on the design of turbomachine blades: extreme operating environments, tight manufacturing tolerances and minimal blade / casing clearances are all commonplace in modern aircraft engine designs [1]. As turbomachines approach these extremes it is important to be confident of the robustness of a design to its service and safety specifications. The effects of uncertainties become especially important to understand for turbomachines operating in these limits of performance (e.g. [2]).

A purely experimental approach is prohibitively expensive and comes too late in the design process, so there is a need for efficient and practical computational models that can predict turbomachine behaviour. The development of such models is mainly limited by two factors: (1) the possibility of non-linear contact between the blades and the surrounding casing structure, and (2) the uncertainties inherent in the contact properties (e.g. the exact geometry of imperfections or the constitutive law of the contact itself).

The presence of non-linearities precludes the use of well-developed linear methods. The two dominant alternatives have their own limitations: case-by-case transient analysis [3] can be prohibitively slow for complex systems, and methods for approximating periodic solutions such as the harmonic balance method [4] can suffer problems with convergence and accuracy for non-smooth nonlinearities such as blade / casing contact [5]. In order to account for uncertainties a Monte Carlo based approach is usually employed [6] which substantially increases an already significant computational load.

There are a wide variety of methods available for modelling uncertainty, which fall into two broad categories: probabilistic and non-probabilistic (e.g. [7]). Probabilistic methods typically characterise unknown quantities in terms of a probability density function (either an assumed distribution or empirically estimated). The effect of these unknowns are propagated to the system outputs, which for non-linear systems necessitates a computationally expensive Monte Carlo analysis. The computational load can be reduced by astute choice of the random variable samples (e.g. importance sampling [8]), but this is nevertheless a costly approach. Nonprobabilistic methods typically represent uncertainties by allowing parameters to be defined in terms of a range of values, e.g. by fuzzy arithmetic [9]. Again, to propagate these uncertainties to the desired system outputs requires calculating the full non-linear response for a large number of cases, which can be prohibitively slow.

The above methods are typically used to handle 'data' or 'parametric' uncertainty: unknown quantities within a chosen model. However, it is often the case that the model itself is uncertain: for a turbomachine blade the contact law and geometry is uncertain. For an interesting discussion on 'model' and 'data' uncertainty see Legault [10].

In this paper an approach is presented based on an adaptation of anti-optimisation [11]. In its usual form, anti-optimisation is used to seek the worst case response of a structure using a non-probabilistic representation of uncertain parameters. The method has also been used to seek the worst case response of a building due to uncertain loading [12] with bounded excitation energy. This study adapts the method to model both 'data' and 'model' uncertainty associated with the non-linear contact and applies it to the vibration analysis of a turbomachine blade undergoing blade / casing contact.

The paper is organised as follows: the chosen test system is first described, followed by an overview of the adapted anti-optimisation approach. A benchmark time-marching model is then presented, which allows validation by a Monte Carlo study. The method is then used to 


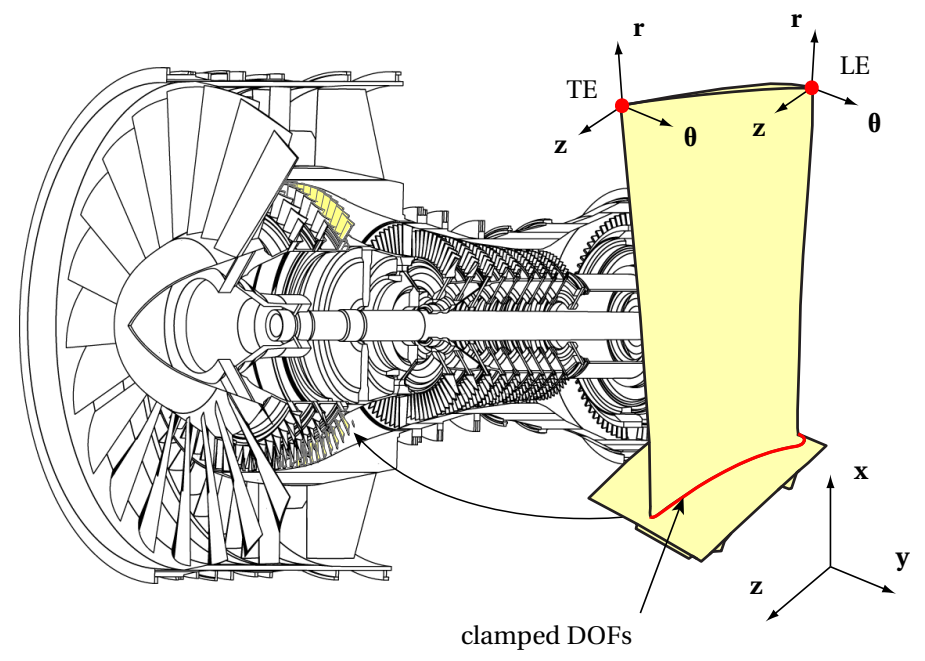

Figure 1: Cut-view of an aircraft engine and zoom over the blade of interest.

analyse the worst-case performance of a blade design in order to demonstrate how the proposed approach could be incorporated into the design phase of a turbomachine. Finally, conclusions are presented that highlight the strengths and weaknesses of the proposed method.

\section{MODELLING}

Note: for the sake of confidentiality, all frequencies are normalised with respect to the first eigenfrequency of the blade at rest.

This study focuses on the dynamic analysis of a single blade from the last stage of the low pressure compressor of an aircraft engine as illustrated in Fig. 1.

\section{Casing profile}

Non-linear contact is assumed to be induced by a misalignment between the casing structure and the blade shaft. This results in a nominal clearance profile $c$ as a function of angular displacement around the casing $\theta$ :

$$
c(\theta)=c_{0} \cos (\theta)+c_{1},
$$

where the constants are chosen to be $c_{0}=1 \mathrm{~mm}$ and $c_{1}=0.95 \mathrm{~mm}$. Figure 2 shows the clearance profile as a function of $\theta$ for a smaller value of $c_{1}$ for the sake of clarity. Later in this study, an ensemble of randomly generated casing profiles will be considered.

\section{Blade}

The axis of rotation of the blade is perfectly rigid. The blade is modelled by a finite element mesh with approximately 52,000 degrees of freedom (DOFs), and is clamped around its root in such a way that the disk dynamics are also neglected. These simplifying hypotheses are justified by experimental observations made in [13].

For this initial study it is assumed that contact can only occur at the leading edge (LE) and at the trailing edge (TE) of the blade tip as depicted in Fig. 1. Also, friction is neglected meaning 


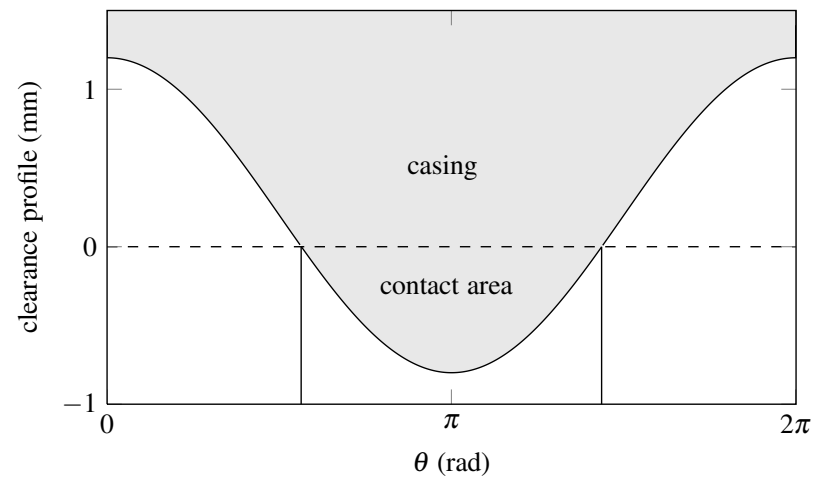

Figure 2: Blade/casing clearance at rest for $c_{0}=1 \mathrm{~mm}$ and $c_{1}=0.2 \mathrm{~mm}$. The dashed line shows the position of the blade tip at rest.

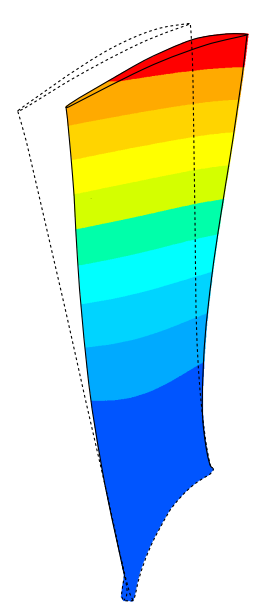

(a) $1 \mathrm{~F}, \omega=\omega_{1}=1$

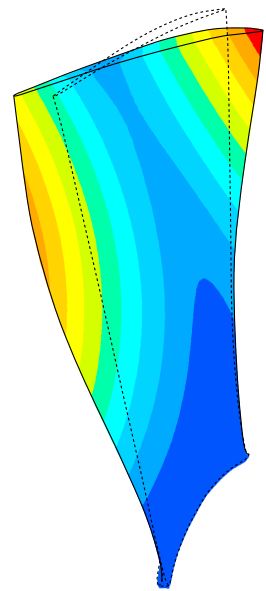

(b) $1 \mathrm{~T}, \omega=4.10$

Figure 3: First bending (1F) and torsion (1T) modes of the blade

that contact treatment is only managed in the local radial $\mathbf{r}$ direction. Finally, centrifugal stiffening of the structure due to the rotational frequency is neglected. These assumptions reduce the complexity of the model to provide faster analysis and clearer interpretation of results, but are not intrinsic limitations of the proposed method.

Because of the very large number of DOFs, direct time integration of contact simulations would lead to cumbersome computation times. As a consequence, the Craig-Bampton [14] component mode synthesis method is used to reduce the number of DOFs of the blade model. Such a method retains the physical contact nodes, avoiding costly mappings between the finite element and the reduced spaces for contact treatment. The final reduced model of the blade features 87 DOFs and the first twenty free vibration modes of the blade are accurately obtained (eigenfrequency error is less than $1 \%$ with respect to the values obtained with the full finite element model).

The first two modes of the blade are depicted in Fig. 3. This blade design has been used for the simulation of blade/abradable coating interaction in [3]. It was found that it is particularly sensitive to blade/abradable coating interaction around several critical frequencies. The intention here is to use the anti-optimisation method to explore whether this behaviour is predicted 
for uncertain contact conditions, without using costly time integration simulations over the rotational frequency range.

\section{Forced response}

It is useful to describe the linear dynamics of the blade in terms of a transfer function matrix relating displacements $\mathbf{u}_{i}$ and forces $\mathbf{f}_{i}$ at the contact nodes:

$$
\left[\begin{array}{c}
u_{r 1} \\
u_{\theta 1} \\
u_{z 1} \\
u_{r 2} \\
u_{\theta 2} \\
u_{z 2}
\end{array}\right]=\mathbf{G}(\omega)\left[\begin{array}{c}
f_{r 1} \\
f_{\theta 1} \\
f_{z 1} \\
f_{r 2} \\
f_{\theta 2} \\
f_{z 2}
\end{array}\right],
$$

where subscripts $r, \theta, z$ denote the radial, lateral and axial components of displacement or force, and 1,2 respectively identify the leading edge and trailing edge contact nodes.

The transfer function matrix $\mathbf{G}(\omega)$ is readily computed from the reduced mass, stiffness and damping matrices (M, K, D respectively) of the reduced order model. Figure 4 shows two example transfer functions $\mathrm{G}_{r 1, r 1}(\omega)$ (blue) and $\mathrm{G}_{r 2, r 2}(\omega)$ (cyan), which are the driving point responses in the radial direction at the leading and trailing edge contact nodes.

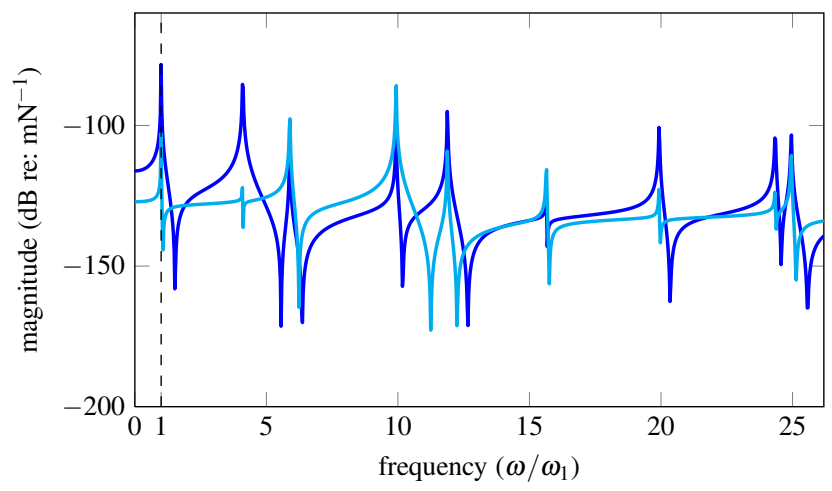

Figure 4: Example driving point transfer functions of the blade: leading edge (blue line) and trailing edge (cyan line).

The first bending mode is identified by the dashed line at $\omega / \omega_{1}=1$ and the torsion mode occurs at $\omega / \omega_{1}=4.1$ (see Fig. 3). It can be seen that the radial compliance of the trailing edge is generally greater than the leading edge for low frequencies (below $5 \mathrm{kHz}$ ). Although the operating blade rotation frequencies are only in the range $\omega_{1} / 20$ to $\omega_{1} / 2$, impacts with the casing structure excite high frequency blade dynamics.

\section{APPLICATION OF ANTI-OPTIMISATION FRAMEWORK}

\section{Method summary}

The blade of interest is illustrated in Fig. 1 and non-linear contacts may occur at the leading and trailing edge. An output metric is denoted M, chosen on the basis of the desired output 
from predictions. Rather than to specify a constitutive law for the non-linearity stemming from contact, the model can be formulated as a constrained optimisation problem: seek the worst metric $\mathrm{M}$ by varying the non-linear forces as if they were independent external loads, subject to constraints which capture basic properties of the non-linear behaviour that are known. Formally:

$$
\begin{array}{ll}
\text { Maximise: } & \mathrm{M}\left(\mathbf{f}_{1}, \mathbf{f}_{2}\right) \\
\text { subject to: } & \mathbf{g}\left(\mathbf{f}_{1}, \mathbf{f}_{2}\right)=0 \\
& \mathbf{h}\left(\mathbf{f}_{1}, \mathbf{f}_{2}\right) \leq 0,
\end{array}
$$

where $\mathrm{M}$ is a scalar metric, $\mathbf{f}_{i}$ is the non-linear force vector on contact nodes $i$ ( $i=1,2$ for this test system), $\mathbf{g}$ is a vector of equality constraints and $\mathbf{h}$ is a vector of inequality constraints. In the following discussion, the appropriate choice of $\mathbf{M}, \mathbf{g}$ and $\mathbf{h}$ will be considered. The focus of this paper is to develop appropriate metrics and constraints: the optimisation itself is solved using the Sequential Quadratic Programming algorithm of Matlab's standard optimisation function fmincon [15]. Development of optimisation algorithms falls beyond the scope of the present study.

In its typical form, anti-optimisation seeks the worst case response by varying model parameters subject to bounds: in contrast the free variables here are time samples of the nonlinear force itself subject to constraints which have a physical interpretation with respect to the underlying non-linear law.

This approach is thought to be particularly well-suited to the vibration analysis of blade / casing contact because:

- uncertainties associated with the non-linearity are captured intrinsically;

- the localised nature of the non-linearities limits the size of the search space of the antioptimisation;

- the method requires careful identification of the desired output from the model (by requiring identification of a target metric $\mathrm{M}$ );

- computing the target metric only requires calculating the solution to a linear forced response problem.

The choice of target metric and constraints are key to the success of the model: different kinds of 'worst case' solutions will be identified for different metrics, and the stringency of the constraints will govern how conservative worst-case predictions are.

\section{Target metric}

There are several outputs that are of interest from a model of turbomachine blade dynamics, for example: peak stress (in order to predict blade failure), root mean square blade displacement (as a measure of the overall vibration level of the blade) or peak blade-tip displacement (to assess the wear profile of abradable coatings). For the purposes of this study, the target metric $\mathrm{M}$ is chosen to be the peak displacement at the contact nodes:

$$
\mathrm{M}=\max \left\|\mathbf{u}_{i}\right\|
$$


where $\mathbf{u}_{i}$ is the displacement at contact nodes $i$ (with $i=1,2$ ). While any of the above metrics could be chosen, peak tip displacement is straightforward and efficient to calculate and provides a clear proof-of-concept demonstration of the anti-optimisation approach.

Note that although the anti-optimisation procedure is intended to find the worst case solutions, the best case can be identified extremely easily by minimising instead of maximising M.

\section{Non-linearity constraints}

The anti-optimisation constraints are chosen to represent what is known about the physical behaviour of the non-linearities. This provides a flexible approach: loose constraints can be used to describe large uncertainties while increasingly stringent constraints can be applied as more information is known. The choice of constraints is central to the model and represents one of the key challenges.

A trade-off exists between choosing constraints that are entirely based on physical arguments without regard for their numerical tractability, and a numerically convenient choice that is sufficient to bound the problem but is not physically derived. The approach taken here is to make an initial choice that has a direct physical interpretation, then to relax them slightly to improve numerical robustness.

The nominal non-linearity described by Eq. (1) is based on an assumed misalignment between the shaft and the centre of a circular casing: this results in a sinusoidal variation in nominal clearance, such that contact must occur during part of the blade revolution. For the purposes of this study it is hypothesised that the exact geometry of the casing profile is uncertain and that the contact law is also uncertain. The following set of constraints is chosen to represent this ensemble of non-linearities (though the choice is by no means unique):

1. The tip radial displacement cannot exceed the nominal clearance profile shown in Fig. 2:

$$
\mathbf{u}_{i} \cdot \mathbf{r} \leq c(\theta)
$$

2. The contact forces can only be in the radial direction (no friction):

$$
\mathbf{f}_{i} \cdot \boldsymbol{\theta}=\mathbf{f}_{i} \cdot \mathbf{z}=0
$$

3. Only compressive contact forces can be applied:

$$
\mathbf{f}_{i} \cdot \mathbf{r} \leq 0
$$

4. The non-linear contact forces can only be non-zero when the blade tip is within an active $z$ one, i.e. within a certain distance $d$ from the nominal casing profile (to represent a range of geometric uncertainty and detailed contact law uncertainty):

$$
\left\|\mathbf{f}_{i}\right\|=0 \text { if } \mathbf{u}_{i} \cdot \mathbf{r} \leq c(\theta)-d,
$$

where $\mathbf{r}, \boldsymbol{\theta}$ and $\mathbf{z}$ are unit vectors corresponding to the local coordinate system at the contact nodes.

These constraints are summarised graphically in Fig. 5 with number labels corresponding to constraints 1-4 above. Note that no specific form of constitutive law is defined for the nonlinearity and that the above constraints are designed to represent a general ensemble of possibilities. In addition, note that the constraints 'close the loop' of the model as they are functions of both the non-linear forces and the tip displacements. 


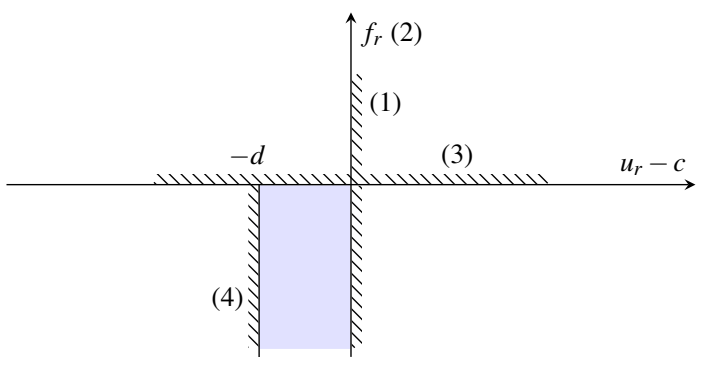

Figure 5: Constraints defining admissible region of $f_{r}-u_{r}$ plane: numbered labels correspond to constraint equations (1)-(4).

\section{Model Reduction}

There are too many variables to carry out a full optimisation in a reasonable time scale: choosing 128 time samples per revolution, the total number of degrees of freedom is 768 (with 6 contact degrees of freedom). This can be significantly improved by using a reduced set of basis functions for the non-linear forces. Some care is needed as the choice of basis implicitly introduces an extra constraint which is not derived from physical arguments. In addition, the set of basis functions needs to be compatible with the physical constraints described above.

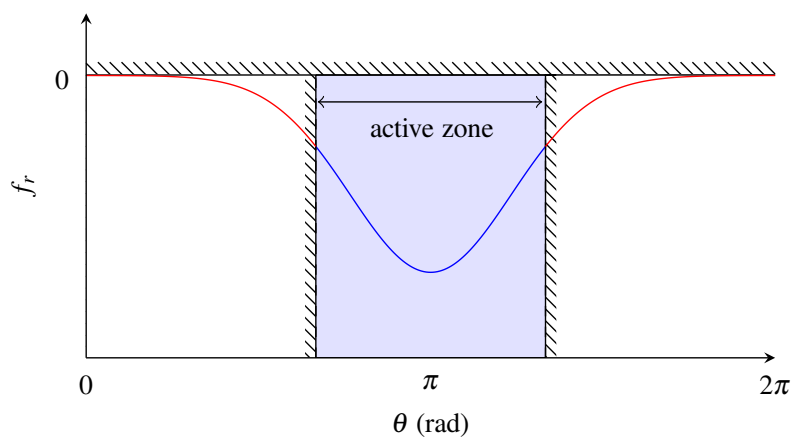

Figure 6: Discontinuous constraints defining admissible region of $f_{r}-\theta$ plane: incompatible with continuous basis functions.

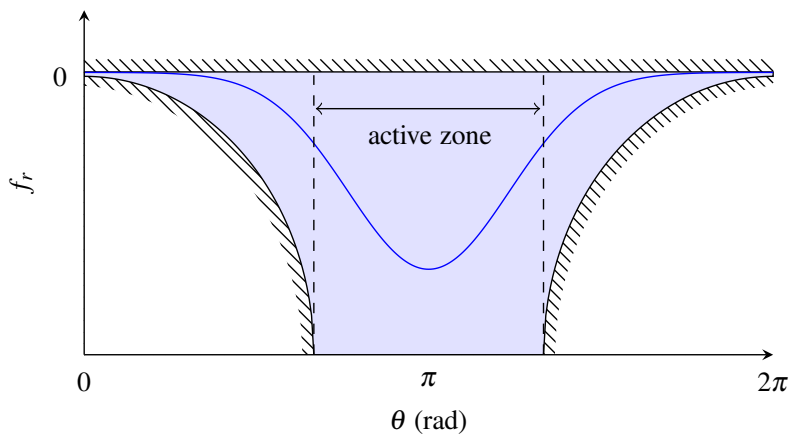

Figure 7: Relaxed constraints defining admissible region of $f_{r}-\theta$ plane.

There is a fundamental difficulty: if the chosen basis functions are continuous they can 


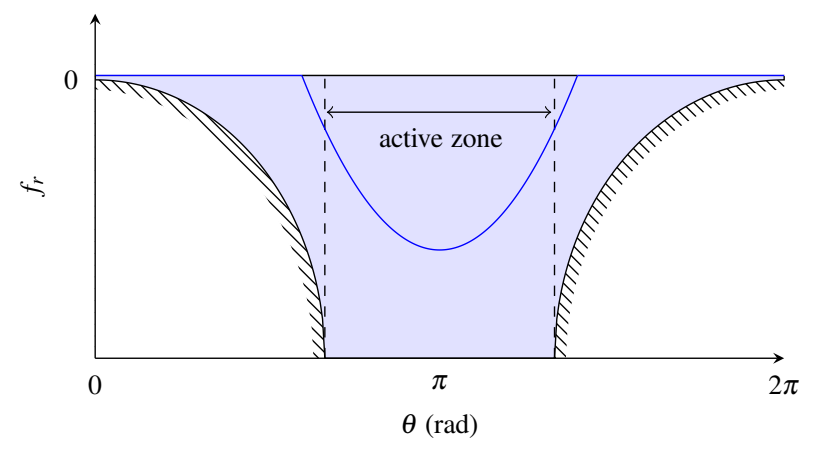

Figure 8: Relaxed constraints defining admissible region of $f_{r}-\theta$ plane with saturation of basis functions.

never be compatible with discontinuous constraints. The problem is most clearly illustrated in reference to Fig. 6 which shows example constraints on radial force $f_{r}$ as a function of $\theta$ for a typical displacement response. Consider limiting non-linear force solutions to consist of a constant plus a purely sinusoidal term. It is clear that no combination exists that satisfies the constraints because the constraints are discontinuous. This problem can be partly solved by relaxing constraint (4) such that it is not discontinuous, as illustrated in Fig. 7. The modified constraint is chosen to allow arbitrary amplitude force when the radial displacement is within the contact zone, and a decreasing amplitude outside of this range. The modified definition of constraint (4) is chosen to be

$$
\left\|\mathbf{f}_{i}\right\| \leq \frac{\mathrm{A}}{c(\theta)-u_{r}(\theta, t)-d} \text { if } c(\theta)-u_{r}(\theta) \geq d,
$$

where $\mathrm{A}$ is a constant that controls the strictness of the constraint (as $\mathrm{A} \rightarrow 0$ the modified constraint tends to the original discontinuous constraint), $c(\theta)$ is the nominal clearance profile, and $u_{r}(\theta, t)$ is the radial component of the contact node displacement. The constant A was chosen empirically, such that the allowable force outside the active zone was negligible.

The relaxation of constraint (4) nevertheless places a too great restriction on the allowable solutions, particularly if only a few harmonics are to be used for the reduced set of basis functions. This was solved by implementing the compression constraint by saturating the basis functions, rather than requiring the linear combination of basis functions to remain negative. This is illustrated in Fig. 8, which shows the effect of saturation on a function consisting of a constant plus a single harmonic. This implementation permits discontinuous solutions that can be arbitrarily large for a finite duration, allowing the representation of impact-type forces that would be expected in reality.

The combination of relaxing constraint (4) and implementing constraint (2) by saturation was found to provide a highly effective compromise between physical realism and numerical robustness. Finally, the scheme was implemented by solving the optimisation problem using only two harmonics, then using that solution as a starting point for a new optimisation with four harmonics, and repeating this procedure using eight harmonics.

\section{BENCHMARK TIME-DOMAIN SOLUTION}

The predictions obtained from the anti-optimisation strategy described above are compared with a time-domain benchmark model. These simulations are carried out based on the strategy 
presented in [16], that was applied in [3] and is briefly recalled here for the sake of clarity. For a definition of variables see the Nomenclature at the end of this paper.

The time-marching procedure involves the explicit central differences scheme combined with a Lagrange multiplier based contact algorithm [17]. At each time step $n$, the procedure is divided into four steps:

1. prediction at time step $n+1$ of the displacements $\mathbf{u}$ :

$$
\mathbf{u}^{n+1, p}=\left[\frac{\mathbf{M}}{h^{2}}+\frac{\mathbf{D}}{2 h}\right]^{-1}\left(\left(\frac{2 \mathbf{M}}{h^{2}}-\mathbf{K}\right) \mathbf{u}^{n}+\left(\frac{\mathbf{D}}{2 h}-\frac{\mathbf{M}}{h^{2}}\right) \mathbf{u}^{n-1}\right)
$$

2. determination of the gap function and detection of the contact nodes of the blade that penetrated the casing.

3. correction of the predicted displacements to ensure a vanishing of the gap function:

$$
\mathbf{g}^{n+1}={ }^{{ }_{\mathbf{N}}}{ }^{\mathrm{t}} \mathbf{u}^{n+1, c}+\mathbf{g}^{p}=\mathbf{0} .
$$

Lagrange multipliers (or contact forces) and updated displacements are obtained as follows:

$$
\left\{\begin{array}{l}
\boldsymbol{\lambda}=\left({ }_{\mathbf{N}}^{\mathrm{t}}\left[\frac{\mathbf{M}}{h^{2}}+\frac{\mathbf{D}}{2 h}\right]^{-1} \cdot \mathbf{v}\right)^{-1} \mathbf{g}^{p} \\
\mathbf{u}^{n+1}=\mathbf{u}^{n+1, p}+\left[\frac{\mathbf{M}}{h^{2}}+\frac{\mathbf{D}}{2 h}\right]^{-1} \cdot{ }_{\mathbf{N}} \boldsymbol{\lambda}
\end{array}\right.
$$

\section{4. time increment.}

In order to remain consistent with the theoretical framework adopted for the anti-optimisation procedure, contact is only treated in the radial direction.

\section{VALIDATION}

\section{Case study}

Before carrying out a full uncertainty study to validate the method, it is interesting to consider the kind of solutions that are obtained by the anti-optimisation approach and compare these with their counterpart benchmark simulations. Two rotational frequencies are considered:

1. $\omega=\omega_{1} / 20$ : this frequency choice represents an almost quasi-static case,

2. $\omega=\omega_{1} / 1.95$ : close to a peak response of the non-linear system.

The nominal casing profile defined in Eq. (1) is used to initiate contact for the benchmark solution. The anti-optimised solution represents the worst case in the presence of uncertainties defined by the constraints described above.

The anti-optimisation approach predicts the upper bound for the steady-state response, so these predictions should be compared with the benchmark solutions after transients have decayed. Therefore the benchmark solution was computed for $\mathrm{N}=100$ blade revolutions with the blade initially at rest. Figure 9 shows the radial displacement at the leading and trailing 


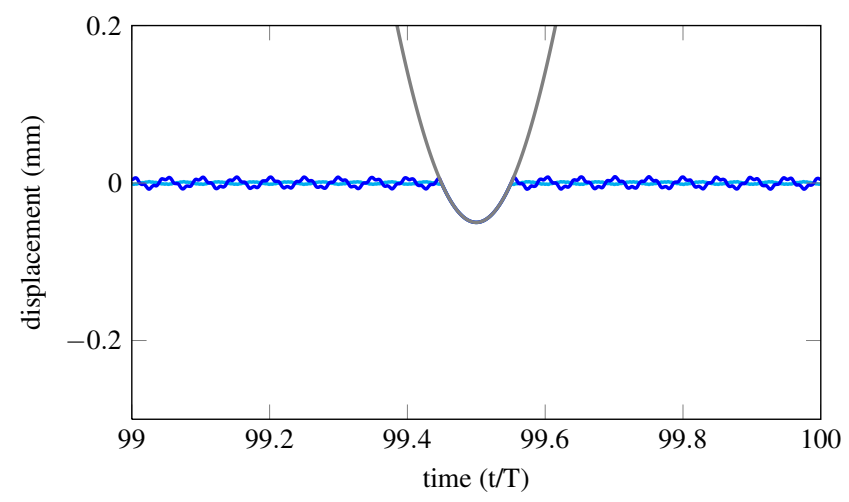

Figure 9: Last period of the benchmark solution at $\omega=\frac{\omega}{20}$.

edge contact nodes (solid blue and cyan lines respectively) for the last period with $\omega=\omega_{1} / 20$, together with the casing profile (grey line). To assess whether steady-state conditions have been reached, the correlation $\mathrm{C}(n)$ of the $n^{\text {th }}$ period with the last period can be quantified by computing the inner product of the normalised vectors:

$$
\mathrm{C}(n)=\frac{\mathbf{u}_{r}(n) \cdot \mathbf{u}_{r}(\mathrm{~N})}{\left\|\mathbf{u}_{r}(n)\right\|_{2}\left\|\mathbf{u}_{r}(\mathrm{~N})\right\|_{2}} .
$$

For this test, $\mathrm{C}(\mathrm{N}-1)=0.99995$ (by definition $\mathrm{C}(\mathrm{N})=1$ ) and the final period represents a reliable indication of the steady-state.

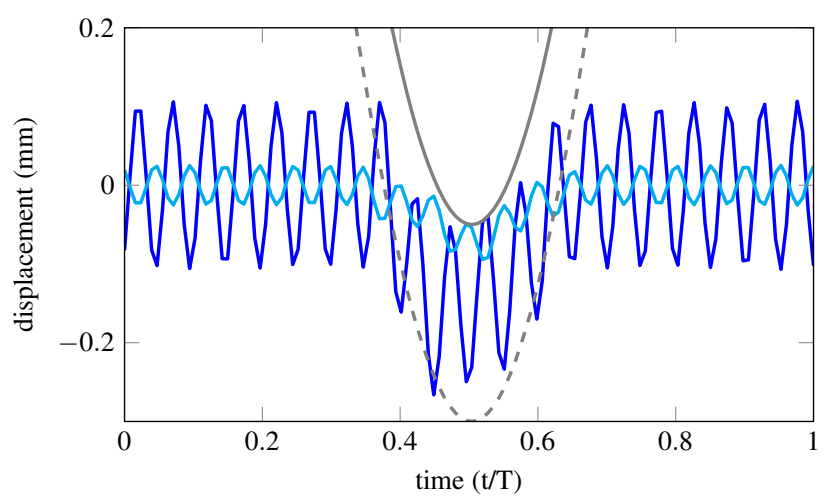

Figure 10: Anti-optimised solution at $\omega=\frac{\omega_{1}}{20}$.

Figure. 10 shows the anti-optimised solution (axes to the same scale as Fig. 9). It is reassuring that the worst-case blade displacement is much greater than the benchmark. Comparing overall maximum displacement amplitudes gives the metrics $\mathrm{M}=0.21 \mathrm{~mm}$ (benchmark) and $\mathrm{M}=1.23 \mathrm{~mm}$ (anti-optimisation). The much larger displacement predicted by the antioptimisation approach is not surprising as it accounts for an ensemble of non-linearities defined by the constraints in Eqs. (5), (6), (7) and (8). The active zone boundary is indicated by the dashed line in Fig. 10: recall that non-linear forces can be non-zero when the radial displacement is between the dashed and solid grey lines.

Figure. 11 shows the last period of the blade response at $\omega=\omega_{1} / 1.95$. In this case the correlation of the previous period with the final period is $\mathrm{C}(\mathrm{N}-1)=0.987$, indicating that the solution 


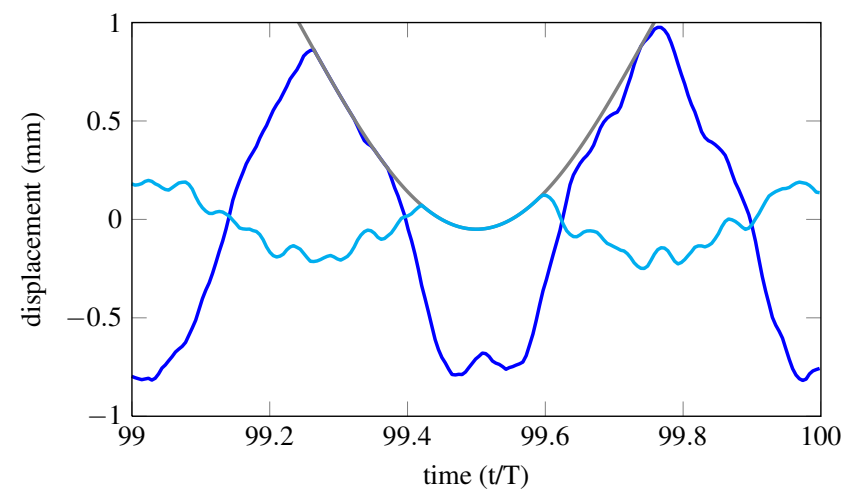

Figure 11: Last period of the benchmark solution at $\omega=\frac{\omega_{1}}{1.95}$.

is again very close to steady-state conditions. The response is much larger than for $\omega=\omega_{1} / 20$ with maximum displacement amplitude of $M=5.89 \mathrm{~mm}$ for the benchmark simulation. The period of the solution is half that of the blade revolution which approximately corresponds to the first bending mode of the structure. The peak occurs at $\omega_{1} / 1.95$ rather than at exactly $\omega_{1} / 2$ due to contact stiffening.

The anti-optimised solution is shown in Fig. 12 and is remarkably similar to the benchmark simulation. In this case the anti-optimised metric $\mathrm{M}=4.9 \mathrm{~mm}$, which is lower than the benchmark. This discrepancy is likely to be precisely because the solutions shown in Figs. 11 and 12 are so similar. The worst case solution is given by the contact geometry of the benchmark simulation, which represents an extreme within the ensemble of non-linearities that can be represented by the constraints (5), (6), (7) and (8). However, there is an additional constraint due to the reduced basis functions chosen. Hence the worst case that can be achieved by antioptimisation is slightly lower than the 'true' bound. This could be solved by a slackening of the constraints to encompass a broader ensemble.

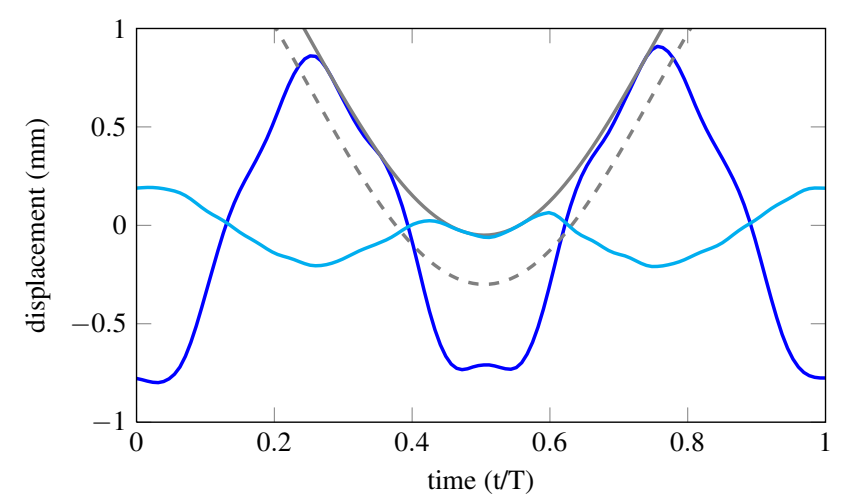

Figure 12: Anti-optimised solution at $\omega=\frac{\omega_{1}}{1.95}$.

The anti-optimisation approach is computationally efficient. Each benchmark solution takes between 6 minutes and 1 hour to solve (for 100 blade revolutions), compared with 13 minutes for the anti-optimisation solution (using the same computing resources). However, the primary advantage of the anti-optimised solution is that it also accounts for uncertainty. 


\section{Monte Carlo simulations}

In order to assess the validity of the anti-optimisation method, a Monte Carlo test was carried out using the benchmark time-domain model. The anti-optimisation constraints could represent uncertainty about the underlying constitutive law of the contact (e.g. contact stiffness variation) or about the contact geometry (or both). For simplicity, only the casing profile is randomised for the present Monte Carlo validation. For a given test, the profile was randomly generated from an ensemble that was consistent with the anti-optimisation constraints. Casing profiles were chosen from:

$$
c(\theta)=c_{0}+\sum_{i=1}^{\mathrm{P}} c_{i} \cos \left(i \theta+\phi_{i}\right)
$$

where $c_{0}=0.825 \mathrm{~mm}$ (i.e. a constant offset in the middle of the active zone, $c_{1}=1 \mathrm{~mm}$ (as before), and the magnitude $c_{i}$, phase $\phi_{i}$ and maximum perturbation order $\mathrm{P}$ are randomised for each test such that the overall casing profile falls within the active zone. A set of profiles is illustrated in Fig. 13: the black and green profiles indicate upper and lower bounds (which are consistent with the active zone defined by Eq. (8)), and the grey profiles are example random profiles: note that some profiles are dominated by short wavelength perturbations while others have a longer wavelength perturbation. The angular velocity of the blade was randomised in the range $0.05<\omega / \omega_{1}<0.52$ (representative of realistic angular velocities).

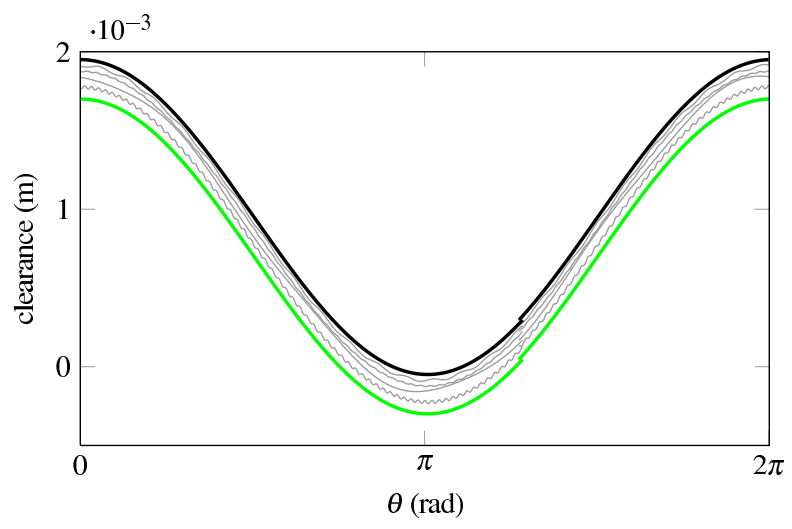

Figure 13: Domain of randomisation of the casing profile.

The time-domain benchmark solutions are carried out over 25 blade rotations. In order to remain consistent with the anti-optimisation procedure, only the simulations for which a steady state was reached - defined here as $\mathrm{C}(\mathrm{N}-1)>0.9$ - are considered in this section.

Figure. 14 shows a summary of the results: 2,300 grey dots represent the target metric $\mathrm{M}$, defined in Eq. (4), for each time simulation (maximum of the amplitude of the displacements of both the leading and trailing edge from the last period of the benchmark simulation with a randomised casing profile). Black and green lines represent benchmark simulations with perfectly sinusoidal casing profiles, which correspond to black and green profiles in Fig. 13.

It can be seen in Fig. 14 that the green casing profile generally gives close to the highest amplitude displacements, while the black casing profile usually results in the minimum. In reference to Fig. 13 this comes as little surprise as these two profiles represent two extremes of the ensemble. This pattern is not followed at the peaks along the frequency sweep: it can be seen 
that there are peaks just above $\omega=\omega_{1} / n$ with $n=2,3,4 \ldots 8$. The peaks are at slightly higher frequencies than the exact resonance subharmonics due to contact stiffening (the presence of the non-linear contact for part of the cycle results in an apparent increase in system stiffness). This effect is strongest for the green profile as the severity of the contact is increased.

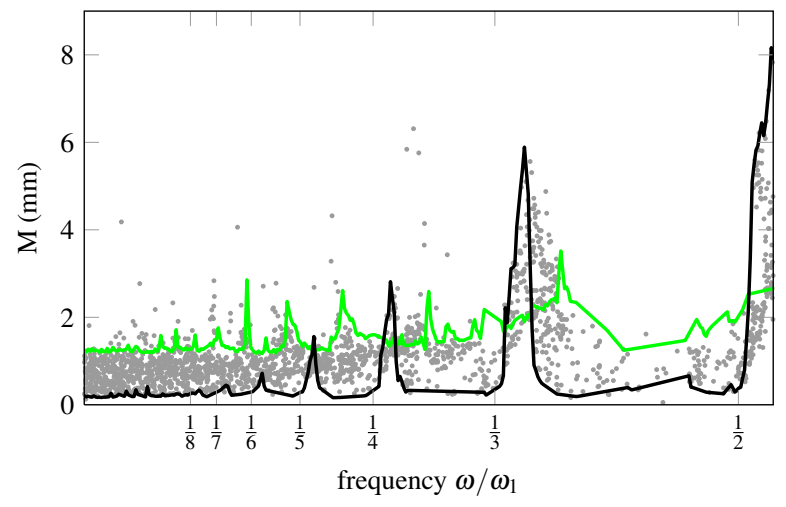

Figure 14: Values of M from Monte Carlo simulations.

Figure. 15 shows the anti-optimisation predictions (red lines) superimposed on the Monte Carlo benchmark results. Both upper and lower bounds are shown (worst and best case predictions respectively). It is very encouraging that the anti-optimised solution predicts the upper bound for almost all of the benchmark Monte Carlo simulations: only $3.6 \%$ of benchmark results give a higher metric $M$.

These results put into perspective the case studies conducted in the previous section. At $\omega=\omega_{1} / 20$, the anti-optimised solution (red line in Fig. 15) was well above the benchmark (black line in Fig. 14). At $\omega=\omega_{1} / 1.95$, the anti-optimised prediction was $20 \%$ below the benchmark. The discrepancy was because the worst case solution was given by the limit of the ensemble of profiles, which could not be attained by the anti-optimisation approach in practice. It can be seen that most discrepancies correspond to peaks of the two extreme profiles (peaks of the black and green lines in Fig. 14): for each of these the discrepancies are small.

The worst case prediction also reveals the underlying trend of the uncertain response with respect to frequency, with non-linear peak responses just above each subharmonic of the first bending mode $\left(\omega=\omega_{1} / n\right.$ for $n=2,3,4 \ldots 8$ ) in agreement with the Monte Carlo data. The predictions are not overly conservative, giving tight bounds across the frequency range studied. The best case predictions (red lower bound in Fig. 15) also agree very well with the Monte Carlo data. The total computation time for the worst case analysis was approximately 5 hours, compared with 120 hours for the Monte Carlo study.

\section{CONCLUSION}

This study focuses on an adapted anti-optimisation procedure for the study of blade vibration arising from contact with the casing. Both data and model uncertainty can be accounted for by using constraints to flexibly describe an ensemble of non-linearities. A case study allows for an in-depth description of the proposed strategy and underlines both its limitations and potential. While the predictions require careful interpretation, their consistency with time simulations is demonstrated. 


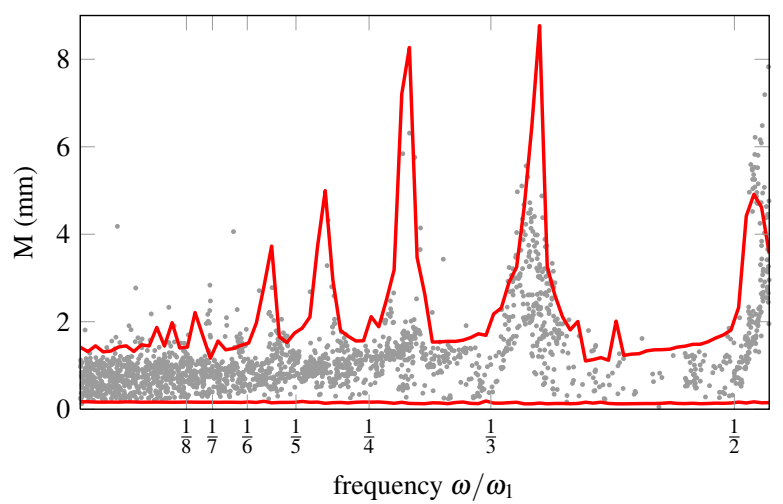

Figure 15: Superposition of the anti-optimised prediction with Monte Carlo simulations.

The consistency of the proposed approach with respect to time simulations is reinforced by comparing worst case predictions with large scale Monte Carlo simulations throughout the rotational frequency range of interest for a specific blade design. Excellent agreement is obtained with worst case predictions accounting for $96 \%$ of the Monte Carlo simulations, without being overly conservative. In addition, the method predicts the trend of the Monte Carlo results.

The anti-optimised results need to be treated with care: no guarantee can be made that global worst cases have been identified. Therefore the anti-optimisation analysis should be repeated with different initial conditions in order to check the robustness of solutions. Nevertheless, this proof-of-concept study demonstrates that the method has a great deal of potential for the efficient analysis of blade / casing interaction in the presence of uncertainties, and the results demonstrate how the proposed approach could be integrated within a blade design optimisation loop.

\section{ACKNOWLEDGEMENT}

Thanks go to Prof. Legrand for hosting Dr. Butlin in the Structural Dynamics and Vibration Laboratory (McGill University) and to Snecma for its technical and financial support.

\section{NOMENCLATURE}

$\lambda \quad$ Lagrange multiplier (contact force)

N contact matrix accounting for normal constraints

D damping matrix

f force vector

g gap function

K stiffness matrix

M mass matrix

u displacement vector 


$\begin{array}{ll}\omega & \text { rotational frequency } \\ \omega_{1} & \text { first eigenfrequency of the blade } \\ \theta & \text { angular position on the casing } \\ \mathrm{t} & \text { indicates a transposed matrix } \\ c & \text { superscript referring to a correction } \\ n & \text { superscript referring to the time step } \\ p & \text { superscript for predicted quantities } \\ \mathrm{C}(n) & \text { correlation factor } \\ c_{i}, \phi_{i} & \text { casing randomisation parameters } \\ d & \text { active zone } \\ h & \text { time step } \\ \mathrm{M} & \text { target metric }\end{array}$

\section{REFERENCES}

[1] R.E. Peacock. A review of turbomachinery tip gap effects: Part 2: rotating machinery. International Journal of Heat and Fluid Flow, 4(1):3-16, 1983. DOI:10.1016/0142-727X(83)90019-X. 2

[2] F. Montomoli, M. Massini, and S. Salvadori. Geometrical uncertainty in turbomachinery: Tip gap and fillet radius. Computers \& Fluids, 46(1):362-368, 2011. DOI:10.1016/j.compfluid.2010.11.031. 2

[3] A. Batailly, M. Legrand, A. Millecamps, and Garcin F. Numerical-experimental comparison in the simulation of rotor/stator interaction through blade-tip/abradable coating contact. Journal of Engineering for Gas Turbines and Power, 134, 2012. DOI:10.1115/1.4006446. 2, 4,10

[4] L. Salles, L. Blanc, F. Thouverez, and A.M. Gouskov. Dynamic analysis of fretting-wear in friction contact interfaces. International Journal of Solids and Structures, 48(10):15131524, 2011. DOI:10.1016/j.ijsolstr.2011.01.035. 2

[5] S. Jones and M. Legrand. The wavelet-Galerkin method for solving PDE's with spatially dependent variables. In Proceedings of the $19^{\text {th }}$ International Congress on Sound and Vibration, Vilnius, Lithuania, July 2012. OAI:hal-00719744. 2

[6] R. Bladh, M.P. Castanier, and C. Pierre. Reduced order modeling and vibration analysis of mistuned bladed disk assemblies with shrouds. Journal of Engineering for Gas Turbines and Power, 121(3):515-523, 1999. DOI:10.1115/1.2818503. 2

[7] B. Mace, K. Worden, and G. Manson. Uncertainty in structural dynamics. Journal of Sound and Vibration, 288(3):423-429, 2005. DOI:10.1016/j.jsv.2005.07.014. 2 
[8] G.I. Schuëller and R. Stix. A critical appraisal of methods to determine failure probabilities. Structural Safety, 4:293-309, 1987. DOI:10.1016/0167-4730(87)90004-X. 2

[9] F. Massa, A. Leroux, B. Lallemand, T. Tison, F. Buffe, and S. Mary. Fuzzy vibration analysis and optimization of engineering structures: Application to Demeter satellite. pages 57-69, 2009. . 2

[10] J. Legault, R.S. Langley, and J. Woodhouse. Physical consequences of a nonparametric uncertainty model in structural dynamics. Journal of Sound and Vibration, 331:5469-5487, 2012. DOI:10.1016/j.jsv.2012.07.017. 2

[11] I Elishakoff and M Ohsaki. Optimization and anti-optimization of structures under uncertainty. Imperial College Press, 2010. 2

[12] C.P. Pantelides and S.-R. Tzan. Convex Model for Seismic Design of StructuresI: Analysis. Earthquake engineering and Structural Dynamics, 25:927-944, 1996. DOI:10.1002/(SICI) 1096-9845(199609)25:9<927::AID-EQE594>3.0.CO;2-H. 2

[13] A. Millecamps, J.F. Brunel, P. Dufrénoy, F. Garcin, and M. Nucci. Influence of thermal effects during blade-casing contact experiments. In Proceedings of the ASME IDETC/CIEDETC2009-86842, San Diego, USA, September 2009. 3

[14] R.R. Craig and C.C. Bampton. Coupling of substructures for dynamics analyses. AIAA Journal, 6(7):1313-1319, 1968. 4

[15] J. Nocedal and S.J. Wright. Numerical Optimization. Springer, 2006. 6

[16] M. Legrand, A. Batailly, B. Magnain, P. Cartraud, and C. Pierre. Full three-dimensional investigation of structural contact interactions in turbomachines. Journal of Sound and Vibration, 331(11):2578-2601, 2012. DOI:10.1016/j.jsv.2012.01.017. 10

[17] N. Carpenter, R. Taylor, and M. Katona. Lagrange constraints for transcient finite element surface contact. International Journal for Numerical Methods in Engineering, 32:103-128, 1991. DOI:10.1002/nme.1620320107. 10 